\title{
Implantation d'un système de conseillers pédagogiques dans un milieu de formation en médecine familiale : une recherche-action
}

\author{
Implementation of an educational adviser system in a family medicine \\ teaching unit: an action research
}

\section{Luc Côtét ${ }^{1}$, Norma Bélanger ${ }^{1}$, Johanne Blais ${ }^{1}$, Marie Desmartis ${ }^{2}$ et Ann MonTreuil ${ }^{1}$}

1 Membres du Département de médecine familiale et de médecine d'urgence de l'Université Laval et enseignants à l'Unité de médecine familiale de l'hôpital Saint-François d'Assise (UMF-SFA) du Centre Hospitalier Universitaire de Québec (CHUQ), Canada

2 Professionnelle de recherche au Centre de recherche du CHUQ, Hôpital Saint-François d'Assise, Canada

Manuscrit reçu le 19 décembre 2007; commentaires éditoriaux formulés aux auteurs le 14 avril 2009; accepté pour publication le 28 mai 2009

\begin{abstract}
Mots clés :
Résumé - Contexte : En 2005, une équipe d'enseignants en médecine familiale à l'université Conseiller pédagogique ; Laval (Québec) a implanté un système jumelant chaque résident à un conseiller pédagogique tutorat; médecine familiale; recherche-action pour la durée de la résidence. La fonction de conseiller était celle d'un guide accompagnant un résident dans son parcours de formation au moyen d'une relation interpersonnelle privilégiée et institutionnalisée. But : Décrire l'implantation du système de conseillers pédagogiques dans un milieu de formation en médecine familiale. Méthodes : Une recherche-action s'est déroulée de juin 2005 à juin 2006 avec la participation des conseillers pédagogiques et des résidents. Le recueil des informations s'est fait au moyen de questionnaires écrits individuels, de groupes de discussion et d'observation des réunions pédagogiques régulières des cliniciens enseignants. Les questionnaires et les groupes de discussion ont fait l'objet d'une d'analyse de contenu thématique. Résultats : Au cours de l'année d'implantation, les conseillers pédagogiques ont pu mieux définir et s'approprier leur nouvelle fonction. L'accompagnement continu du résident dans son développement professionnel et les conseils spécifiques en regard de besoins particuliers sont devenus les spécificités de cette fonction. La triangulation des sources d'information et des méthodes de recueil des données a permis d'enrichir les résultats. Globalement, la recherche-action a positivement contribué à l'implantation du changement pédagogique dans ce milieu. Conclusion : La clarification de la fonction de conseiller pédagogique, son appropriation et l'application de moyens concrets permettant de la mettre en pratique sont les principales retombées de cette étude. La recherche-action a permis d'offrir des lieux de réflexion et d'échanges dans l'équipe d'enseignants et entre les enseignants et les résidents en plus d'aider à trouver des solutions aux obstacles rencontrés dans l'implantation du système.
\end{abstract}




\section{Keywords:}

Educational adviser; mentorship; family medicine; action research
Abstract - Context: In 2005, a group of clinical teachers in family medicine at Laval University (Québec) implemented a system assigning an educational adviser to each resident for the duration of the residency. The adviser's role was to guide the resident along his or her professional path by means of institutionalized and privileged relationship. Objective: To describe the implementation of an educational adviser system in a family medicine teaching unit. Methods: An action research was undertaken between June 2005 and June 2006 with the collaboration of educational advisers and residents. Data were collected from written individual questionnaires, focus groups and observations completed during regular educational meetings of clinical teachers. Questionnaires and focus groups were analyzed thematically. Results: During the implementation year, educational advisers have better clarified and adopted their new role. The continuous guidance regarding resident's professional development as well as its specific needs became specificities of educational advisers roles. Sources of information and data collection methods triangulation have enriched results. In general, the action research has positively contributed to the implementation of an educational change in this setting. Conclusion: The clarification and the assumption of the educational adviser's role and the application of concrete means to allow its practice are the main contributions of this study. The action research has promoted reflective practice and discussions within the teaching team and between teachers and residents. In addition, it helped to solve problems encountered during the implementation system.

\section{Introduction}

En 2004, le comité de révision du programme universitaire de médecine familiale de l'université Laval (Québec, Canada) recommandait l'implantation d'un système de conseillers pédagogiques auprès des résidents (internes) du programme et diffusait un document aux cliniciens enseignants. Le rôle du conseiller pédagogique, tel qu'il est conçu dans le cadre de ce programme, est d'accompagner le résident dans le but de l'aider à développer une pratique réflexive et à construire son expertise et son identité professionnelle. Ce concept semble très voisin de celui de tuteur, dont font état certains auteurs en France, notamment dans le cadre de la formation délivrée au cours du diplôme d'études spécialisées de médecine générale ${ }^{[1]}$. Ce système visait à « accompagner le résident dans son cheminement dans le programme en permettant l'émergence et la consolidation des attitudes nécessaires à la pratique de la médecine familiale » ${ }^{[2]}$. Plus spécifiquement, étaient visés, grâce à un accompagnement personnalisé, le développement de l'identité professionnelle du résident par le biais d'un appui longitudinal (sur
24 mois) ainsi que l'élaboration de stratégies préventives pour l'aider à faire face aux situations de stress inhérentes à la formation. Dans ce contexte, il semblait opportun que le conseiller assure régulièrement l'échange de la rétroaction formative et sommative en lien avec les apprentissages du résident.

La pertinence de ce système reposait sur l'importance du tutorat pédagogique et du soutien professionnel dans une perspective de continuité, aspects jugés préoccupants dans le programme antérieur. La fonction de conseiller pédagogique devenait donc celle d'un guide accompagnant un résident dans son parcours de formation au moyen d'une relation interpersonnelle privilégiée et institutionnalisée. Pour appliquer cette recommandation, le comité de révision du programme proposait que chaque résident soit jumelé de façon aléatoire à un conseiller pédagogique dès son entrée dans le programme.

En 2005, l'équipe d'enseignants de l'unité de médecine familiale de l'hôpital Saint-François d'Assise (UMF-SFA) du centre hospitalier universitaire de Québec implantait le système en jumelant chaque résident à un clinicien-enseignant-conseiller pour la 
durée de la résidence. Toutefois, même si l'importance de la fonction de guide auprès des résidents ne soulevait en elle même aucune réserve chez les membres de l'équipe, plusieurs s'interrogeaient sur la pertinence globale d'un tel système. Leur questionnement soulevait les problématiques suivantes :

- la difficulté à concilier cette nouvelle fonction avec celle de superviseur que le clinicien enseignant assumait depuis plusieurs années. Les tâches, en supervision, sont centrées sur la gestion de la qualité des soins et sur la rétroaction à échanger en lien avec les apprentissages ;

- les préoccupations quant aux impacts potentiels d'un appariement aléatoire, d'autant que le résident n'était pas impliqué dans le choix de son conseiller ;

- les questions soulevées par le fait de concilier les fonctions de conseiller et celle d'évaluateur, surtout en contexte sommatif. Agir en tant que conseiller exigeait, pour plusieurs, le développement d'une relation de confiance, relation essentielle au partage authentique par le résident de ses intérêts, ses doutes et inquiétudes, ainsi que de ses stress. Par opposition, le superviseur est responsable des évaluations formatives et sommatives du résident; cette responsabilité requiert la plus grande objectivité possible, ce qui faisait dire à plusieurs qu'il y avait conflit d'intérêt entre les deux fonctions.

C'est dans ce contexte que les enseignants ont décidé d'implanter le système en y intégrant une démarche de recherche-action à laquelle ils ont, dès le départ, associé les résidents. Puisque l'implantation du système était un changement pédagogique important, car il modifiait les pratiques du milieu, ils croyaient que le fait d'impliquer tous les acteurs contribuerait positivement à la mise en place du système et aiderait à en tirer tout le potentiel. D'ailleurs, les données de la littérature accréditent de façon consensuelle la notion que l'une des conditions principales requises pour que les processus de changement produisent les résultats attendus est que les personnes visées par le changement se l'approprient afin d'y donner un sens ${ }^{[3,4]}$.
Revue de la littérature

Nous avons procédé à une revue méthodique mais non exhaustive de la littérature en éducation médicale consacrée au thème de l'accompagnement pédagogique personnalisé. Pour ce faire, dans le cadre de notre recherche documentaire, nous avons interrogé les données de la base bibliographique Medline avec les mots-clés «conseiller pédagogique, mentor, tuteur, modèle, clinicien enseignant, superviseur précepteur et enseignement clinique ». Parallèlement, nous avons sélectionné des articles à partir de références provenant de divers écrits que nous avions déjà sur ces thèmes. Le Dictionnaire actuel de l'éducation ${ }^{[5]}$ a aussi servi de source d'information.

En éducation médicale, il y a une certaine confusion entre des fonctions voisines et souvent distinctes, dénommées par des appellations différentes (conseiller pédagogique, mentor, superviseur, modèle de rôle, précepteur, tuteur, etc.). Ces concepts sont souvent présentés indifféremment (par exemple : clinicien enseignant, superviseur et précepteur clinique), parce que perçus comme désignant des fonctions équivalentes ; à l'inverse, ils sont parfois proposés comme désignant des fonctions distinctes et complémentaires découlant du rôle de clinicien enseignant ${ }^{[6-9]}$. Quoi qu'il en soit, des finalités communes caractérisent habituellement ces fonctions : a) le soutien au développement des compétences professionnelles, particulièrement l'expertise clinique; b) l'accompagnement du développement de l'identité professionnelle; c) le soutien du développement personnel inhérent au développement professionnel; d) le développement d'un lien de confiance à l'intérieur d'une relation hiérarchique.

Toutefois, certaines distinctions doivent être faites. Il apparaît que le mentor est une personne d'expérience guidant et supportant un novice dans son développement professionnel au cours d'une relation qui dure habituellement quelques années. La relation mentor-protégé est explicite au sens où les deux parties s'engagent volontairement dans cette relation privilégiée ${ }^{[10-14]}$. Il y a donc un choix explicite et délibéré qui s'effectue. Quant au modèle de 
rôle (role model), il s'agit d'une personne admirée en raison de sa manière d'être et de se comporter ${ }^{[15]}$. À la différence du mentor, cette relation n'est habituellement pas explicite. En effet, un clinicien enseignant ne se présente pas auprès d'un résident en lui disant : «Observe-moi attentivement car je suis ton modèle !». Ainsi, un modèle peut exercer une influence positive sur quelqu'un sans en être conscient et sans même connaître cette personne. Cependant, certains avantages à l'exploitation optimale du rôle de modèle par le superviseur ont été identifiés. Dans ce contexte, le superviseur doit non seulement posséder des attributs significatifs, comme clinicien, comme enseignant et comme personne, mais aussi être conscient de ce rôle et le jouer de manière explicite, en tirant tout le potentiel de la démonstration comme stratégie pédagogique ${ }^{[15-17]}$.

Pour Saarikoski ${ }^{[10]}$, le superviseur intègre trois fonctions principales : l'enseignement, l'évaluation (formative et sommative) et le mentorat (ou le support). Dans cette perspective, le système de conseillers pédagogiques est une stratégie éducative complémentaire au système de supervision habituel en médecine familiale, dans lequel les résidents sont en contact avec plusieurs superviseurs pendant leur formation.

\section{But et objectifs spécifiques de la recherche}

Le but du présent travail est de décrire l'implantation du système de conseillers pédagogiques à l'UMFSFA et plus spécifiquement de : 1) décrire l'évolution des représentations des conseillers et des résidents quant aux fonctions et tâches du conseiller pédagogique ; 2) répertorier les avantages et les inconvénients du système pour les conseillers et pour les résidents ; 3) identifier les obstacles émergeant en cours d'implantation et appliquer des solutions s'y rapportant.

\section{Méthodes}

Nous avons choisi de réaliser une recherche-action de type collaboratif dans laquelle un groupe de professionnels décide d'examiner ensemble et de façon critique leur pratique (ici, l'implantation du système de conseillers pédagogiques), de rechercher des moyens de l'améliorer et de contribuer à la résolution d'une problématique qu'ils partagent ${ }^{[18]}$. La recherche-action est une méthode de recherche qualitative reposant sur le postulat que l'implication des personnes dans la définition d'un problème et dans la recherche de solutions pour le résoudre est préalable au changement des pratiques. Elle se caractérise par des cycles itératifs de planification, d'action, d'observation et de réflexion au fur et à mesure de l'évolution du processus ${ }^{[19]}$.

\section{Recueil des informations}

Les informations ont été recueillies auprès de deux sources. Tous les cliniciens-enseignants jouant le rôle de conseiller pédagogique $(n=14)$, tous les résidents de première année $(n=12)$ et six résidents de deuxième année ont volontairement participé à l'étude.

Des questionnaires écrits, des groupes de discussion et l'observation des réunions pédagogiques ont été utilisés comme méthodes de collecte de données. La diversification (triangulation) des sources d'information et des méthodes pour les recueillir figure parmi les critères de crédibilité (validité interne) en recherche qualitative ${ }^{[20,21]}$. Le recueil des informations s'est fait à trois reprises entre juin 2005 et juin 2006 : au début de l'année (lors de l'arrivée de nouveaux résidents), au milieu (octobre-novembre 2005) et à la fin (avril-juin 2006). En fin d'étude, à l'aide d'une échelle de type Likert à six niveaux ( $1=$ très inconfortable à $6=$ très confortable), nous avons demandé individuellement aux conseillers de situer leur niveau de confort dans l'exercice de leur fonction de conseiller pédagogique, au début et en fin de projet.

\section{Les questionnaires écrits}

Des questionnaires individuels à réponses ouvertes ont été élaborés par l'équipe de recherche. Six questions ont été rédigées en lien avec les objectifs de 
la recherche, à savoir les perceptions du rôle de conseiller, les tâches réalisées en cours d'implantation du système, les avantages et inconvénients du système, les obstacles rencontrés ainsi que les facteurs ayant favorisé l'adhésion au système. Ces questionnaires ont été complétés de manière anonyme par les conseillers pédagogiques (début et fin de l'étude) et par les résidents (début, milieu et fin de l'étude).

\section{Les groupes de discussion focalisée (focus groups)}

Les groupes de discussion visaient à enrichir la représentation individuelle et collective du rôle de conseiller pédagogique et à développer des modalités d'intervention pour optimiser le système. Le plan des rencontres comportait, dans l'ordre, les points suivants : un rappel des objectifs de la recherche, la synthèse des réponses aux questionnaires, une période de questions et de discussion visant à identifier des actions à maintenir ou à entreprendre pour implanter le système. Le guide d'animation utilisé lors de ces groupes a été élaboré par l'équipe de recherche. Chaque rencontre a été enregistrée en audio, avec le consentement des participants.

Cinq rencontres de groupe, d'une durée d'environ une heure trente chacune, ont eu lieu durant l'année, selon l'échéancier suivant :

- deux groupes de discussion distincts (l'un avec les conseillers, l'autre avec les résidents) au début de l'étude;

- deux groupes distincts (l'un avec conseillers, l'autre avec résidents), au milieu de l'étude;

- un groupe conjoint (conseillers et résidents), à la fin de l'étude.

\section{L’observation des réunions pédagogiques}

Les réunions pédagogiques existent depuis plusieurs années à l'UMF. Elles regroupent tous les superviseurs, médecins et autres professionnels, et ont lieu deux fois par mois, à raison d'une durée de deux heures chacune. Elles visent à recueillir l'information requise pour documenter l'évaluation formative et sommative des résidents en stage, partager une rétroaction et assurer le suivi pédagogique. L'observation non participante des réunions pédagogiques par une professionnelle de recherche, anthropologue de formation, avait pour but de mieux comprendre comment s'intégrait le nouveau système dans la pratique pédagogique des enseignants de l'UMF. Pendant ces rencontres, la professionnelle notait ses observations dans un journal de bord, souvent sous forme d'incidents critiques (ex : un conseiller pédagogique relatant une difficulté vécue lors de la rencontre avec son résident).

\section{Analyse des informations}

Les entretiens effectués dans le cadre des groupes de discussion ont fait l'objet d'une transcription intégrale sous forme de verbatim. Une analyse de contenu thématique des réponses aux questionnaires écrits et des verbatim des groupes de discussion a été réalisée selon les étapes reconnues pour ce type d'analyse ${ }^{[22]}$. Le contenu manifeste fut codifié à partir d'un procédé de catégorisation d'où émergent progressivement, et jusqu'à saturation, les thèmes et les catégories de l'analyse de contenu. Les réponses aux questionnaires et les verbatim des groupes de discussion ont d'abord été analysés par la professionnelle de recherche, puis validés par l'ensemble des chercheurs. Tous les chercheurs ont contribué à la synthèse finale de l'analyse. Le fait d'impliquer plusieurs chercheurs à différentes étapes de l'analyse (triangulation des chercheurs) augmente la validité du processus ${ }^{[20,21]}$. Enfin, de par la nature même de la recherche-action, les participants ont pu vérifier la justesse des analyses car celles-ci leur étaient présentées lors des groupes de discussion. Cette vérification par les participants (member checking) contribue à augmenter la crédibilité en recherche qualitative.

\section{Résultats}

Fonctions et tâches du conseiller pédagogique

Les questionnaires individuels ont permis de recueillir et de comparer les perceptions des 
Tableau I. Fonctions et tâches du conseiller pédagogique perçues par les résidents et les conseillers pédagogiques.

\begin{tabular}{|l|c|c|c|c|}
\hline \multicolumn{1}{|c|}{ Fonctions et tâches } & \multicolumn{2}{c|}{ Résidents } & \multicolumn{2}{c|}{ Conseillers pédagogiques } \\
\hline & $\begin{array}{c}\text { Au début } \\
n=18\end{array}$ & $\begin{array}{c}\text { À la fin } \\
n=17^{*}\end{array}$ & $\begin{array}{c}\text { Au début } \\
n=14\end{array}$ & $\begin{array}{c}\text { À la fin } \\
n=13^{*}\end{array}$ \\
\hline Remise des évaluations et rétroactions constructives & 13 & 13 & 12 & 13 \\
\hline $\begin{array}{l}\text { Personne-ressource pendant la résidence/Conseil pour les choix } \\
\text { de stage/ Orientation/ Information }\end{array}$ & 14 & 7 & 13 & 10 \\
\hline Soutien pédagogique ou psychologique au résident & 9 & 3 & 5 & 7 \\
\hline Pratique d'examens oraux & 1 & 3 & & 2 \\
\hline Démarches ponctuelles (ex : lettre de recommandation) & & & & 2 \\
\hline
\end{tabular}

* Un résident et un conseiller pédagogique n’ont pas répondu au questionnaire écrit en fin d'étude.

participants au début et à la fin d'étude quant aux fonctions et tâches du conseiller pédagogique (Tableau I).

\section{Le point de vue des résidents}

Au début de l'étude, les résidents attribuaient trois principales fonctions et tâches au conseiller pédagogique : 1) répondre à leurs questions sur la résidence et la pratique future; 2) remettre les évaluations périodiques et partager une rétroaction constructive; 3) les soutenir dans leur cheminement, autant au plan pédagogique que psychologique. À la fin, la remise des évaluations et l'échange de rétroactions constructives caractérisaient la contribution du conseiller, alors que ses fonctions de personneressource et de soutien étaient moins rapportées. Cependant, lors des groupes de discussion, la majorité des résidents ont réitéré l'importance du conseiller en tant que personne-ressource, surtout au plan pédagogique :

«Il y a des objectifs à atteindre en deux ans pour devenir un bon médecin de famille, et si on a l'occasion de discuter avec quelqu'un qui nous connaît, je trouve ça important qu'il nous dirige, qu'il nous conseille en lien avec les choses de base que nous devons faire. »

\section{Le point de vue des conseillers pédagogiques}

Au début, les réponses aux questionnaires montrent que les conseillers avaient sensiblement la même perception que les résidents quant à la contribution du conseiller (remise des évaluations et personneressource). Toutefois, lors du premier groupe de discussion (sans les résidents), il est apparu que les fonctions du conseiller n'étaient pas aussi claires que les réponses au questionnaire le laissaient entendre, pour les deux raisons suivantes :

- L'ambiguiité entre les fonctions du conseiller pédagogique et du superviseur :

«Quand j'ai regardé les résultats, ce qui m'a le plus étonné, c'est l'ambiguïté du rôle : un conseiller pédagogique, est-ce que c'est la même chose qu'un bon superviseur? Dépendamment de comment on perçoit notre rôle de superviseur, on peut y voir un rôle très pointu, fermé versus un rôle plus large incluant le soutien au développement professionnel avec une dimension personnelle là-dedans. »

- La difficulté de formuler une définition unique du conseiller pédagogique, ses fonctions variant selon la nature des interactions :

«Moi je pense que le rôle va probablement varier entre les intervenants et le résident selon ce qui se passe. Avec un résident problématique, on ne vit pas l'interaction de la même façon qu'avec un résident qui fonctionne bien. Ça va être variable selon les circonstances. »

En milieu d'année, ces discussions ont incité les chercheurs à élaborer un Guide du conseiller pédagogique regroupant trois fonctions principales, des exemples de tâches associées à chacune de ces fonctions, ainsi qu'une proposition de modalités d'application (Annexe 1). Ce guide a été remis à tous les 
Tableau II. Avantages du système de conseillers pédagogiques pour les résidents, selon les conseillers pédagogiques et les résidents.

\begin{tabular}{|l|c|c|c|}
\hline \multicolumn{1}{|c|}{ Avantages du système pour les résidents } & Conseillers pédagogiques & \multicolumn{2}{c|}{ Résidents } \\
\hline $\begin{array}{l}\text { Identification d'une personne ressource pour transmettre } \\
\text { informations et conseils }\end{array}$ & 10 & $\begin{array}{c}\text { Au début } \\
n=18\end{array}$ & $\begin{array}{c}\text { À la fin } \\
n=17\end{array}$ \\
\hline $\begin{array}{l}\text { Développement de liens privilégiés/de confiance avec } \\
\text { un enseignant }\end{array}$ & 5 & 10 & 9 \\
\hline Meilleur suivi du cheminement du résident & 2 & 9 & 4 \\
\hline Rétroactions au résident & 1 & 3 & 5 \\
\hline
\end{tabular}

* La question se rapportant aux avantages n'apparaissait dans le questionnaire écrit des conseillers qu'en début d'étude.

conseillers et résidents, puis discuté et approuvé par les conseillers.

En fin d'étude, lors du groupe de discussion (conseillers et résidents), les fonctions suivantes ont fait l'objet d'un large consensus, d'autant plus que les conseillers les avaient assumées pendant l'année :

- l'accompagnement continu du résident dans son cheminement, via la remise des évaluations périodiques :

«Une des caractéristiques du conseiller pédagogique, c'est la relation sur une base longitudinale. On ne retrouve pas ça ailleurs. Qu'est ce que le conseiller pédagogique fait qu'un bon superviseur ne fait pas? C'est que sur une période de 24 mois et non de trois ou six mois, il développe une relation unique avec le résident afin de l'aider à devenir un bon médecin de famille. Ça c'est une différence. »

- Les conseils spécifiques en fonction des besoins : «J'ai commencé à jouer le rôle sans trop savoir ce qu'il fallait que je fasse. En plus du suivi longitudinal, j'ai été active en donnant des conseils comme, par exemple, dans le choix des stages et le choix de carrière. Les besoins de mon résident se situaient à ce niveau-là. Maintenant, c'est clair que j'ai un rôle de conseiller.»

\section{Avantages du système pour les résidents}

Au début de l'étude, tous les conseillers (14) et les résidents (18) percevaient que ce système était avantageux pour le résident. À la fin, 15 résidents rapportaient toujours des impacts positifs : le tableau II présente le type d'avantages que représente le système pour les résidents, selon le point de vue des conseillers et des résidents. L'identification d'une personne-ressource pouvant transmettre des informations et des conseils, un meilleur suivi du cheminement du résident et le développement de liens privilégiés / de confiance avec un enseignant sont les principaux avantages rapportés.

Nous avons approfondi les avantages du système pour les résidents lors du dernier groupe de discussion qui réunissait tous les participants à l'étude. Après une première année d'implantation, les principaux avantages rapportés ont été :

- l'identification du conseiller en tant que personne responsable de la remise des évaluations périodiques (formatives et sanctionnelles) et de l'échange de la rétroaction favorisant un meilleur suivi des résidents :

«Moi, j'ai trouvé ça intéressant de recevoir les évaluations et de pouvoir échanger avec la même personne ... J'ai trouvé ça bien agréable par rapport à l'année passée. » (résident en fin de formation)

«... le fil conducteur en termes de suivi de l'évaluation. Ça m'apparaît être le principal avantage... Discuter avec un résident que tu connais plus; je trouvais ça intéressant. » (conseiller) 
Tableau III. Inconvénients du système de conseillers pédagogiques, selon les conseillers pédagogiques et les résidents.

\begin{tabular}{|l|c|c|c|c|}
\hline \multicolumn{1}{|c|}{ Inconvénients } & Conseillers pédagogiques & \multicolumn{2}{c|}{ Résidents } \\
\hline & $\begin{array}{c}\text { Au début } \\
n=14\end{array}$ & $\begin{array}{c}\text { À la fin } \\
n=13\end{array}$ & $\begin{array}{c}\text { Au début } \\
n=18\end{array}$ & $\begin{array}{c}\text { À la fin } \\
n=17\end{array}$ \\
\hline Manque d'affinité conseiller-résident & 6 & 1 & 9 & \\
\hline Variabilité / Inégalité de l'encadrement & 2 & & 3 & \\
\hline $\begin{array}{l}\text { Manque de temps pour les rencontres / problème } \\
\text { de gestion de l'horaire }\end{array}$ & 2 & 2 & 1 & \\
\hline Appropriation de la fonction de conseiller pédagogique & 2 & & & \\
\hline $\begin{array}{l}\text { Conflits entre les fonctions d'évaluateur et de soutien } \\
\text { du conseiller pédagogique }\end{array}$ & & 1 & & 1 \\
\hline
\end{tabular}

- l'identification du conseiller en tant que personneressource accessible, répondant aux questions du résident :

«... travailler avec une personne-ressource facilement accessible. On sait qu'on peut la rencontrer s'il y a des questionnements particuliers à propos de certaines choses. » (résident)

En questionnant les conseillers (questionnaire écrit et groupes de discussion) sur les avantages du système, ceux-ci ont mentionné principalement le développement d'une relation privilégiée avec le résident. Cette relation de confiance était jugée indispensable à l'accompagnement du résident dans son cheminement professionnel. À la fin de l'étude, quelques conseillers ont aussi rapporté que le système avait été pour eux une occasion de mieux saisir la portée des objectifs du programme.

\section{Inconvénients du système et obstacles rencontrés}

En début d'étude, 12 des 14 conseillers pédagogiques et 11 des 18 résidents anticipaient des inconvénients. À la fin de l'étude, quatre conseillers et un résident en percevaient toujours. Le tableau III présente le type d'inconvénients associés à l'implantation du système, tels que perçus par les conseillers et les résidents.

Le problème du manque d'affinité conseillerrésident, inconvénient principal associé au système en début d'étude, n'a été rapporté que par un seul conseiller et par aucun résident à la fin. Parmi les autres inconvénients mentionnés, le manque de temps pour les rencontres ainsi que les conflits entre les fonctions d'évaluateur et de soutien restaient préoccupants pour quelques conseillers.

L'appariement aléatoire a été un thème récurrent lors des groupes de discussion. En fait, il semble que ce type d'appariement inquiète dans l'éventualité d'une relation problématique conseiller-résident :

«...c'est un des aspects qui fait que ce jumelage est complexe... Les responsables du programme de résidence ont décidé que c'était un appariement imposé. Quand ça va bien, il n'y a pas de problème, mais quand ça va mal, qu'est ce qu'on fait? Dans ce cas-là, les résidents sont coincés car la pression hiérarchique joue. » (conseiller)

Lors d'une discussion de groupe avec les résidents, les avantages et les inconvénients de l'appariement imposé ont été comparés à ceux de l'appariement par affinité. $11 \mathrm{y}$ a eu consensus quant au fait qu'il serait difficile d'appliquer l'appariement par affinité dans le contexte actuel, puisque les nouveaux résidents ne connaissent pas les enseignants quand ils arrivent à l'UMF. Par ailleurs, dans les situations où il $\mathrm{y}$ aurait un problème d'affinité, le conseiller pédagogique peut toujours se limiter à discuter les évaluations avec le résident, 
ce-dernier pouvant rencontrer un autre enseignant pour répondre à d'autres besoins :

«Essayer de faire un appariement selon les affinités, ça semble extrêmement compliqué et c'est probablement impossible. Pour que ce soit faisable, il faudrait attendre plusieurs mois afin que les gens se connaissent. Mais c'est sûr que lorsqu'on a un conseiller pédagogique qui est imposé, je me dis, à la limite, la pire chose qui puisse arriver, si ça ne fonctionne pas, c'est que ton conseiller pédagogique va remettre les évaluations, mais pour tout le reste, le résident va aller voir ailleurs, puis, il va y avoir un appariement naturel qui va se faire. » (conseiller)

Lorsqu'ils ont été invités à s'exprimer sur les obstacles qu'ils avaient rencontrés dans l'application du système (questionnaires et groupes de discussion), peu de conseillers et de résidents en percevaient, à l'exception du manque de temps et des difficultés liées à la planification des rencontres conseiller-résident. Par ailleurs, plusieurs résidents et conseillers ont indiqué que les conflits entre les fonctions d'évaluateur et de soutien du conseiller pédagogique restaient un objet important de préoccupation. À ce sujet, certains conseillers ont fait remarquer qu'ils auraient eu besoin d'une meilleure définition et précision des fonctions et tâches au départ :

«Au début du projet, j'aurais aimé en savoir un peu plus sur les tâches à faire: quelle forme ça allait prendre, la durée de rencontre qu'on devrait prévoir. Le côté pratique quoi! C'est certain que je me suis adapté au cours de l'année. Il faut dire aussi que pour nous, c'est plus clair maintenant ce qu'un conseiller doit faire alors que ça l'était moins quand on a commencé. » (conseiller)

À la fin de l'étude, des résidents restaient préoccupés par les possibles conséquences de discussions avec un conseiller qui est aussi un évaluateur :

«...ceci dit, moi je me fie à leur jugement. Je pense que les superviseurs doivent et sont capables quand ils évaluent les résidents de faire abstraction de ce qui se dit dans leur bureau. Mais je pense que certains résidents s'empêchent de parler de certaines choses avec leur conseiller de peur que ça influence négativement leur évaluation. » (résident)
Évolution du niveau de confort des conseillers

Douze conseillers ont répondu. Le niveau de confort moyen rapporté fut de 3,3 au début et de 4,3 en fin de projet. Lors d'une réunion pédagogique, ces résultats furent présentés aux conseillers. Les principaux éléments rapportés ayant contribué à cette amélioration sont :

- le partage des expériences vécues entre collègues et pour les enseignants novices, l'échange avec des enseignants plus expérimentés ;

- l'officialisation d'une fonction de soutien que plusieurs enseignants assumaient déjà de manière informelle ;

- l'expérience acquise lors de la mise à l'essai du système ;

- la contribution de la démarche de rechercheaction comme moyen d'implanter ce système car elle avait favorisé la pratique réflexive et apporté des modifications concrètes suite aux problèmes rencontrés.

\section{Discussion}

L'implantation d'un changement pédagogique, même s'il semble attrayant à première vue, ne se fait pas sans heurts car il interfère avec la culture et les pratiques d'un milieu. De façon rétrospective, nous constatons que le système de conseillers pédagogiques a véritablement pris racine dans notre milieu. Nous établissons les trois constats suivants concernant la démarche d'implantation qui a été réalisée :

\section{Les conseillers pédagogiques ont pu mieux définir et $s^{\prime}$ approprier leur nouvelle fonction}

Au cours de l'année, nous avons constaté l'évolution de la perception du rôle de conseiller pédagogique et son appropriation progressive chez les enseignants et les résidents. Au début, pour la majorité, la fonction et les tâches du conseiller pédagogique étaient ambigües car elles étaient perçues indistinctement de celles du superviseur. En cours d'année, et particulièrement lors des discussions de groupe, la clarification conceptuelle s'est faite et elle s'est enrichie. 
L'accompagnement continu du résident dans son développement professionnel et les conseils spécifiques en regard de besoins particuliers sont devenus les spécificités de cette fonction. Les stratégies qui ont contribué à ces processus de définition et d'appropriation sont : l'expérimentation du rôle pendant une année, doublée d'un suivi rigoureux de l'évolution du système par la recherche-action ; la référence à un court document écrit, le Guide du conseiller pédagogique (Annexe 1), produit en cours d'étude.

Autant pour les résidents que pour les conseillers, la continuité dans l'encadrement du résident est un des impacts positifs de la mise en place du système. Il est stimulant de constater que cette continuité apparaît comme un avantage majeur et celui-ci est encore présent après trois ans d'implantation. En fournissant aux conseillers une structure propice au développement d'une relation privilégiée avec un résident, le système contribue à ce que les cliniciens enseignants connaissent mieux les résidents qu'ils supervisent, adoptent un point de vue plus global sur leur évolution professionnelle et assurent un meilleur suivi en discutant notamment de leurs besoins. Certains enseignants ont d'ailleurs mentionné que la fonction de conseiller représentait un facteur de valorisation de leur rôle de clinicien enseignant en médecine familiale.

Le principal inconvénient du système, mis en évidence au début de la recherche par les médecins enseignants et par les résidents, est un impact découlant de l'appariement imposé, soit les problèmes possibles d'incompatibilité conseiller-résident. Cependant, même si ce problème a fait l'objet de plusieurs interventions lors des groupes de discussion, il a été rapporté de façon très marginale lors des réponses aux questionnaires à la fin du projet. Ce problème anticipé en début d'année ne s'est donc pas concrétisé.

\section{La triangulation des sources et des méthodes de recueil des informations a contribué à la qualité de la démarche}

Tel que mentionné dans la littérature, la triangulation des sources et des méthodes de recueil des informations contribue à la crédibilité des résultats en recherche qualitative. Le fait d'impliquer les cliniciens enseignants et les résidents de même que la combinaison des trois méthodes a non seulement permis d'approfondir les informations recueillies mais aussi de démontrer la concordance des points de vue. Dans les faits, les questionnaires complétés individuellement ont servi de base aux rencontres de groupe en nous permettant de spécifier les principaux thèmes à explorer. Les groupes de discussion ont joué un rôle déterminant dans l'évolution de l'implantation du rôle de conseiller pédagogique car ils ont permis d'enrichir les différentes représentations de la fonction, tout en stimulant l'expression de plusieurs points de vue, y compris les questions et les doutes. Alors que les premiers groupes ont fourni des informations sur les attentes, les craintes, les doutes reliés au nouveau système, ceux réalisés en cours d'année nous ont permis de cerner les problèmes, d'intervenir pour mettre en place des solutions (ex : la production du Guide), c'est-à-dire d'ajuster l'intervention. Le dernier groupe de discussion a permis en quelque sorte de réaliser une évaluation de groupe de la première année d'implantation formelle du système. Les résidents ont exprimé leur surprise et leur satisfaction de constater que certaines préoccupations exprimées par leurs superviseurs rejoignaient les leurs. De même, les réunions pédagogiques régulières ont permis aux participants d'intégrer ce nouveau rôle en partageant avec leurs collègues leur expérience à partir de situations concrètes.

\section{La recherche-action a positivement contribué à l'implantation du changement pédagogique}

La nature collaborative de la recherche-action a favorisé la clarification et l'appropriation de la nouvelle fonction de conseiller pédagogique et son implantation dans notre milieu. Parce qu'elle a suscité l'engagement des enseignants et des résidents, la recherche-action a été particulièrement utile à tous les participants, en particulier aux enseignants moins expérimentés qui ont pu bénéficier de l'expérience des plus anciens. Cette méthode a été choisie parce 
qu'elle se caractérise par le va-et-vient entre l'action et la réflexion et permet l'ajustement des interventions selon l'évolution du processus. C'est précisément ce qui s'est produit à l'UMF-SFA. D'ailleurs, lors du dernier groupe de discussion, les conseillers et les résidents ont unanimement salué la pertinence du choix de cette méthode.

\section{Les limites de l'étude}

La première limite se situe au niveau de l'analyse des résultats. Même si nous étions conscients de l'importance de la triangulation des chercheurs comme facteur de crédibilité en recherche qualitative (au moins deux chercheurs analysent indépendamment tout le matériel), pour des raisons logistique et de faisabilité, la première analyse a été faite uniquement par la professionnelle de recherche qui, dans un deuxième temps, a soumis son analyse aux autres chercheurs. Idéalement, une analyse indépendante faite par au moins deux chercheurs aurait été préférable. Toutefois, des réunions d'équipe ont eu lieu régulièrement entre les chercheurs pour discuter les analyses, sans oublier la vérification des résultats par les participants lors des groupes de discussion. Ces mesures nous permettent de penser que nos résultats sont crédibles.

L'autre limite a trait à la transférabilité des résultats (généralisation) à d'autres contextes. Cette question est complexe et fait l'objet de fréquents débats dans la littérature en recherche qualitative. Certains auteurs ${ }^{[23]}$ suggèrent qu'il appartient au lecteur de décider si les conclusions d'une étude qualitative font du sens pour lui (résonance) et ce, même si les caractéristiques du milieu où s'est déroulée la recherche sont plus ou moins semblables, voire différentes d'autres contextes. Une autre manière de concevoir la transférabilité est plutôt d'évaluer si les résultats contribuent à l'avancement des connaissances (theorical understanding) d'une problématique à l'étude et non pas de chercher les similitudes entre les contextes, y compris les caractéristiques de la population à l'étude. Quelle que soit la perspective adoptée, nous pensons que le contexte de l'UMFSFA est tout à fait transférable à d'autres contextes de formation en médecine familiale au Québec et au Canada et probablement à certains contextes de formation en médecine générale, en France notamment. De même, l'avancement des connaissances sur la fonction de conseiller pédagogique en contexte de formation en médecine familiale nous semble transférable autant ici qu'ailleurs.

\section{Conclusion}

La clarification de la fonction de conseiller pédagogique, son appropriation et l'application de moyens concrets permettant de la mettre en pratique sont les principales retombées de cette étude. La rechercheaction a permis d'offrir des lieux de réflexion et d'échanges dans l'équipe d'enseignants et entre les enseignants et les résidents en plus d'aider à trouver des solutions aux obstacles rencontrés dans l'implantation du système. Malgré la présence d'obstacles réels, en particulier le temps qu'exige un tel système, nous savons qu'il est viable et utile après plus de trois années d'implantation.

Finalement, le thème du développement de l'identité professionnelle en médecine familiale mériterait une attention particulière. On sait que l'identité professionnelle évolue non seulement au cours des études en résidence mais tout au long de la vie professionnelle. Quelle est notre représentation de l'identité professionnelle, quels sont les facteurs personnels et contextuels associés à sa construction pendant la résidence ? En quoi et comment le rôle de conseiller pédagogique peut-il contribuer à son développement? Voilà quelques-unes des questions qui nous interpellent non seulement dans notre milieu mais aussi dans d'autres UMF et au programme de médecine familiale et qui méritent d'être étudiées dans le futur.

\section{Contributions}

Tous les auteurs ont participé à la conception et à la réalisation de l'étude, de même qu'à l'analyse et à la discussion des résultats. Luc Côté a rédigé la version originale de l'article et tous les auteurs ont contribué à la production de la version finale. 


\section{Approbation éthique et financement}

Cette recherche, approuvée par le Comité d'éthique de la recherche du CHUQ, a été subventionnée par le Collège des médecins de famille du Canada.

\section{Remerciements}

Les auteurs remercient les enseignants et résidents de l'Unité de médecine familiale du Centre Hospitalier Universitaire de Québec (hôpital Saint-François d'Assise).

\section{Annexe 1}

\section{Guide du conseiller pédagogique à l'Unité de médecine familiale Hôpital Saint-François-d'Assise}

\section{A1- Principales fonctions et tâches du conseiller}

\section{Principales fonctions}

1. Être une personne ressource pour le résident dans son cheminement professionnel pendant sa résidence formatives et sommatives lors du stage de médecine familiale
2. Transmettre périodiquement les évaluations

\section{Exemple de tâches}

- Identifier, avec le résident, des moyens pour l'aider à atteindre les objectifs du programme et des stages

- Identifier, avec le résident, ses intérêts professionnels et ses objectifs et le soutenir dans l'atteinte de ces derniers

- Aider à la préparation aux examens, si désiré

- Assurer un suivi aux évaluations du résident, incluant celles dans les stages de spécialités

- Guider / conseiller le résident dans le choix de stages / activités en fonction du type de pratique désiré

- Recueillir les informations nécessaires à l'évaluation du résident lors de son stage de médecine familiale

- Identifier précocement, avec l'aide de l'ensemble des enseignants, les difficultés du résident et les points à améliorer et lui transmettre l'information le plus rapidement possible

- Identifier avec le résident des stratégies d'amélioration

3. Intervenir, au besoin, pour aider le résident à gérer les stress associés à sa formation
- Identifier tôt, avec le résident, les problèmes rencontrés lors des différents stages

- Reconnaître les manifestations de stress excessif, d'épuisement ou de dépression afin de lui suggérer les ressources pertinentes 


\section{Guide du conseiller pédagogique à l'UMF St-François d'Assise (suite)}

\section{A2- Modalités d'application de la fonction de conseiller pédagogique}

A) Planification d'une première rencontre avec le résident dès le début de la résidence, c.-à-d. avant septembre. Les objectifs suggérés sont, entre autres :

- Faire connaissance, recueillir un minimum d'informations sur le parcours du résident (parcours académique), ses caractéristiques et ses perceptions de son futur rôle ;

- Présenter les fonctions et les tâches du conseiller pédagogique;

- Expliquer les moyens utilisés pour lui transmettre une évaluation la plus fidèle de son fonctionnement : réunions pédagogiques et suivi de ces réunions, feuilles « suivi des apprentissages », etc.

- S'entendre sur les modalités de prise de rendez-vous pour les prochaines réunions.

B) Planification du moment d'une deuxième rencontre :

Cette deuxième rencontre sera initiée par le conseiller pédagogique au début du stage de médecine familiale à l'UMF ou avant ce stage, si celui-ci a lieu dans une période trop éloignée (plus de 6 mois après la $1^{\text {ère }}$ rencontre). Les objectifs suggérés sont :

- Discuter du fonctionnement général du résident dans les différents stages et plus spécifiquement lors du stage de médecine familiale;

- Discuter des objectifs du programme (voir cahier du programme);

- Discuter des objectifs personnels de formation du résident.

C) Le nombre et le moment des autres rencontres sont déterminés en fonction des besoins de chaque résident, à l'exception des rencontres formelles d'évaluation. Ces rencontres pourraient permettre de répondre aux différents objectifs identifiés dans le document du programme de médecine familiale Guide du conseiller pédagogique à l'Unité de médecine familiale.

UMF-SFA, Janvier 2006

\section{Références}

1. Le Mauff P, Pottier P, Goronflot L, Barrier J. Évaluation d'un dispositif expérimental d'évaluation certificative des étudiants en fin de troisième cycle de medicine générale. Pédagogie Médicale 2006;7:142-54.

2. Programme de médecine familiale. Guide à l'intention du conseiller pédagogique. Préparer les médecins de famille pour le Québec de demain. Québec : Faculté de médecine, Université Laval. 2004:2 (disponible sur demande auprès des auteurs).

3. Fullan M. Leading in a culture of change. San Francisco: Jossey-Bass, 2001.

4. Bland CJ, Starnaman, S, Wersal L, Moorhead-Rosenberg L, Zonia S, Henry R. Curricular Change in Medical Schools: How to Succeed. Acad Med 2000;75:575-94.

5. Legendre R. Dictionnaire actuel de l'éducation. Montréal : Guérin : 1993.
6. Kilminster SM, Jolly BC. Effective supervision in clinical practice settings: a literature. Review. Med Educ 2000;34:827-40.

7. Kilminster SM, Grant J, Jolly BC. AMEE Guide No. 27: Effective educational and clinical supervision. Med Teach 2007;29:2-19.

8. Sutkin G, Wagner E, Harris I, Schiffer R. What makes a good clinical teacher in medicine? A review of the literature. Acad Med 2008;83:452-66.

9. Bray L, Nettleton P. Assessor or mentor? Role confusion in professional education. Nurse Education Today 2007;27:848-55.

10. Saarikoski M. Mentor relationship as a tool of professional development of student nurses in clinical practice. Int $\mathrm{J}$ Psychiatr Nurs Res 2003;9:1014-24.

11. Swenson JR, Boyle AB, Last J. Mentorship in medical education. Annals RCPSC 1995;28:165-69. 
12. Manek N. Developing coaching skills: A practical approach for education supervision. The Clinical Teacher 2004;1:74-6.

13. Zerzan JT, Hess R, Schur E, Phillips RS, Rigotti N. Making the most of mentors: A guide for mentees. Acad Med 2009;4:140-44.

14. Hayes EF. Approaches to mentoring: How to mentor and be mentored. J Am Acad Nurse Pract 2005;7: 442-45.

15. Côté L, Leclère $H$. How clinical teachers perceive the doctor-patient relationship and themselves as role models. Acad Med 2000;75: 1117-24.

16. Chamberland M, Hivon R. Les compétences de l'enseignant clinicien et le modèle de rôle en formation clinique. Pédagogie Médicale 2005;6:98-111.

17. Cruess SR, Cruess RL, Steinert Y. Role modelling -making the most of a powerful teaching strategy. BMJ 2008; 336: 718-21.

18. Savoie-Zajc L, Bednarz N. Action Research and Collaborative Research: Their specific contributions to professional development. Educational Action Research : An International Journal 2007;15:577-96.
19. Dolbec A. La recherche-action. Dans : Gauthier B. (ed). Recherche sociale : de la problématique à la collecte des données. Québec : PUQ, 1997:467-96.

20. Côté L, Turgeon J. Comment lire de façon critique les articles de recherche qualitative en médecine ? Pédagogie Médicale 2002,3:81-90.

21. Reidy M, Mercier L. La triangulation. Dans Fortin MF. Le processus de la recherche : de la conception à la réalisation. Ville Mont-Royal : Décarie, 1996:318-24.

22. L'Ecuyer R. L'analyse de contenu : notions et étapes. Dans Deslauriers JP. Les méthodes de la recherche qualitative. Sillery : PUQ, 1988:49-65.

23. Kuper A, Lingard L, Levinson, Critically appraising qualitative research. BMJ 2008;337:718-21.

Correspondance et offprints : Luc CÔTÉ, Département de médecine familiale et de médecine d'urgence, Faculté de médecine, Bureau 2476 Pavillon Ferdinand-Vandry, Université Laval, G1K7P4 Québec, Québec, Canada

Mailto : luc.cote@fmed.ulaval.ca 